\title{
Superconductivity in the High- $\boldsymbol{T}_{c} \mathrm{Bi}-\mathrm{Ca}-\mathrm{Sr}-\mathrm{Cu}-\mathrm{O}$ System: Phase Identification
}

\author{
R. M. Hazen, (1) C. T. Prewitt, ${ }^{(1)}$ R. J. Angel, ${ }^{(1)}$ N. L. Ross, ${ }^{(1)}$ L. W. Finger, ${ }^{(1)}$ C. G. Hadidiacos, ${ }^{(1)}$ \\ D. R. Veblen, ${ }^{(2)}$ P. J. Heaney, ${ }^{(2)}$ P. H. Hor, ${ }^{(3)}$ R. L. Meng, ${ }^{(3)}$ Y. Y. Sun, ${ }^{(3)}$ Y. Q. Wang, ${ }^{(3)}$ Y. Y. Xue, ${ }^{(3)}$ \\ Z. J. Huang, ${ }^{(3)}$ L. Gao, ${ }^{(3)}$ J. Bechtold, ${ }^{(3)}$ and C. W. Chu ${ }^{(3)}$ \\ ${ }^{(1)}$ Geophysical Laboratory, Carnegie Institution of Washington, Washington, D.C. 20008 \\ ${ }^{(2)}$ Department of Earth and Planetary Sciences, The Johns Hopkins University, Baltimore, Maryland 21218 \\ ${ }^{(3)}$ Department of Physics, University of Houston, Houston, Texas 77004 \\ (Received 2 February 1988)
}

\begin{abstract}
Four phases are observed in superconducting $\mathrm{Bi}-\mathrm{Ca}-\mathrm{Sr}-\mathrm{Cu}-\mathrm{O}$ samples. The superconducting phase, with onset temperature near $120 \mathrm{~K}$, is a 15.4- $\AA$-layered compound with composition near $\mathrm{Bi}_{2} \mathrm{Ca}_{1} \mathrm{Sr}_{2} \mathrm{Cu}_{2} \mathrm{O}_{9}$ and an $A$-centered orthorhombic unit subcell $5.41 \times 5.44 \times 30.78 \AA^{3}$. X-ray diffraction and electron microscopy data are consistent with a structure of alternating perovskite and $\mathrm{Bi}_{2} \mathrm{O}_{2}$ layers. High-resolution transmission electron microscopy images reveal a $b$-axis superstructure of $27.2 \AA$, numerous (001) stacking faults, and other defects.
\end{abstract}

PACS numbers: $74.70 . \mathrm{Vy}$

The discovery of high-temperature superconductivity in oxide systems with the $\mathrm{K}_{2} \mathrm{NiF}_{4}{ }^{1}$ and $\mathrm{YBa}_{2} \mathrm{Cu}_{3} \mathrm{O}_{7}{ }^{2}$ structures has led to an intensive search for other superconducting oxides. Recently Michel et al. ${ }^{3}$ described superconductivity at temperatures to $22 \mathrm{~K}$ in the $\mathrm{Bi}-\mathrm{Sr}$ $\mathrm{Cu}-\mathrm{O}$ system, and $\mathrm{Chu}$ et al. observed superconductivity up to $120 \mathrm{~K}$ in the multiphase $\mathrm{Bi}-\mathrm{Al}-\mathrm{Ca}-\mathrm{Sr}-\mathrm{Cu}-\mathrm{O}$ and $\mathrm{Bi}-\mathrm{Ca}-\mathrm{Sr}-\mathrm{Cu}-\mathrm{O}$ (BCSCO) systems. ${ }^{4}$ Some samples show only one superconducting transition with a midpoint transition temperature near $90 \mathrm{~K}$, but others display a clear step in both resistive and magnetic measurements with a $T_{c} \approx 115 \mathrm{~K}$ and an onset at $\approx 120 \mathrm{~K}$. $\mathrm{X}$-ray powder patterns do not match any known oxide superconductor structure. In this Letter we identify the 90-K superconducting phase, as well as three coexisting phases. We suggest, furthermore, that the $115-\mathrm{K}$ phase is similar to the $90-\mathrm{K}$ phase with only minor modification-perhaps improved atomic order.

We studied three $\mathrm{BCSCO}$ samples synthesized by solid-state reaction of $\mathrm{Bi}_{2} \mathrm{O}_{3}, \mathrm{CuO}, \mathrm{SrCO}_{3}$, and $\mathrm{CaCO}_{3}$ in air. Details of the process are described elsewhere. ${ }^{5}$ The compositions of the $\mathrm{Bi}: \mathrm{Ca}: \mathrm{Sr}: \mathrm{Cu}$ samples were $1: 1: 1: 1$ for BCSCO- $a$ and BCSCO- $b$, and $1: 1: 1: 3$ for BCSCO- $c$. Heat treatments were different for samples BCSCO $-a$ and $-b$. Bar samples were cut from sintered pellets. A four-lead method was employed for the resistance measurements and a PAR model M155 vibratingsample magnetometer was used for investigating dc magnetization. The temperature dependence of $R$ for these samples appears in Fig. 1.

Only one superconducting transition is observed in BCSCO- $a$, but two occur in BCSCO- $b$ and BCSCO- $c$. dc magnetization measurements show that $12 \%$ of the Meissner effect in these samples is associated with the 115-K transition. The total Meissner effect of the samples is about $40 \%$ compared to a $\mathrm{Pb}$ sample. The partial Meissner effect is attributed to the multiphase nature and low flux trapping of the sample. Greater Meissner effects (up to $\approx 60 \%$ ) are observed by us in other BCSCO samples ${ }^{5}$; in some specimens as much as twothirds of the effect is associated with the $115-\mathrm{K}$ transition.

$\mathrm{X}$-ray powder diffraction patterns of the $\mathrm{Cu}$-rich $\mathrm{BCSCO}-c$ sample revealed a substantial amount of unreacted copper oxide, which was not present in BCSCO- $a$ or $-b$. Samples were examined by optical and electron microscopy, $x$-ray powder and single-crystal diffraction, and electron microanalysis. Powder $x$-ray patterns were taken on a Rigaku model DMAX-3B automated diffractometer. Single-crystal $\mathrm{X}$-ray studies were made on a Rigaku automated four-circle diffractometer with monochromated rotating-anode Mo source. Electron microanalyses were obtained on a JSM model 35 scanning electron microscope, operated at $20 \mathrm{KV}$ and $0.01-\mu \mathrm{A}$ beam current with a $2.5-\mu \mathrm{m}$ spot size. Standards include pure $\mathrm{Bi}$ and $\mathrm{Cu}$ metal, Sr-bearing glass, and a diopside 65 -jadeite 35 glass. We employed a Philips

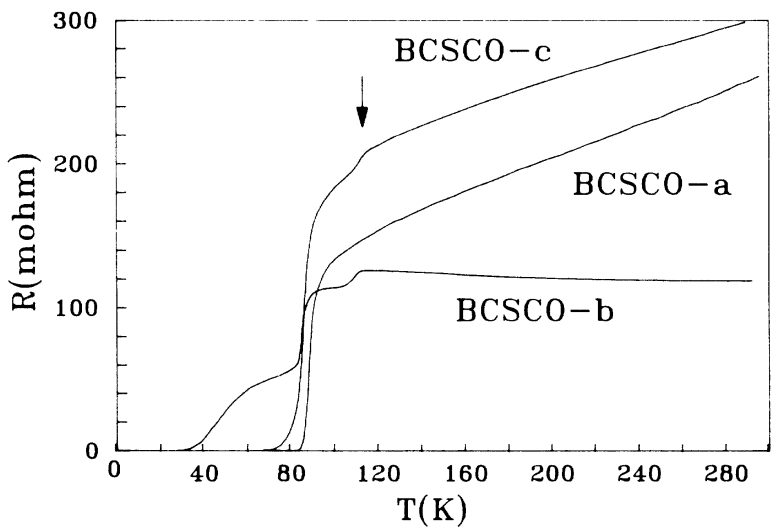

FIG. 1. Temperature dependence of resistance of the Bi$\mathrm{Ca}-\mathrm{Sr}-\mathrm{Cu}$ oxide superconductor. 
model EM420 transmission electron microscope (TEM) with EDAX Si-Li detector and a Princeton GammaTech 4 data analysis system. TEM samples were mounted on holey-carbon, Be-mesh grids to avoid $\mathrm{Cu}$ contamination during analysis.

Four phases in these samples include two alkaline earth copper oxides, a bismuth alkaline earth oxide, and the superconductor. Elongated subhedral to euhedral crystals of a transparent, birefringent, pleochroic (red to colorless) phase are prominent in grain mounts of all three samples examined with a polarizing microscope. Needlelike crystals up to $100 \mu \mathrm{m}$ long, though abundant in some samples, constitute a small fraction (probably $\leq 5 \%$ ) of the sample volume. Electron microanalysis of five different crystals of this insulating phase yields an average composition of $\left(\mathrm{Ca}_{0.92} \mathrm{Sr}_{0.08}\right)_{2}\left(\mathrm{Cu}_{0.96} \mathrm{Bi}_{0.03}\right) \mathrm{O}_{3}$. Some or all of the bismuth may represent background secondary scattering from adjacent Bi-rich phases; therefore, the crystals may be bismuth free. A $90 \times 10 \times 5$ $\mu \mathrm{m}^{3}$ crystal was selected for single-crystal $\mathrm{x}$-ray study and was found to have orthorhombic symmetry with $a=12.234, b=3.777$, and $c=3.257 \AA$. These values are almost identical to those reported for stoichiometric $\mathrm{Ca}_{2} \mathrm{CuO}_{3}$ by Teske and Müller-Buschbaum. ${ }^{6}$

A second bismuth-poor phase, distinguished in grain mounts as black, opaque, elongated euhedral to subhedral grains up to $60 \mu \mathrm{m}$ long, was found in samples $\mathrm{BCSCO}-b$ and $\mathrm{BCSCO}-c$. The average composition is $\left(\mathrm{Ca}_{0.6} \mathrm{Sr}_{0.4}\right) \mathrm{Cu}_{1.75} \mathrm{O}_{3}$, based on microanalysis of four grains. Approximately 0.03 atom of bismuth was also detected per one alkaline earth cation. Three crystals of this phase have been examined by single-crystal $x$-ray

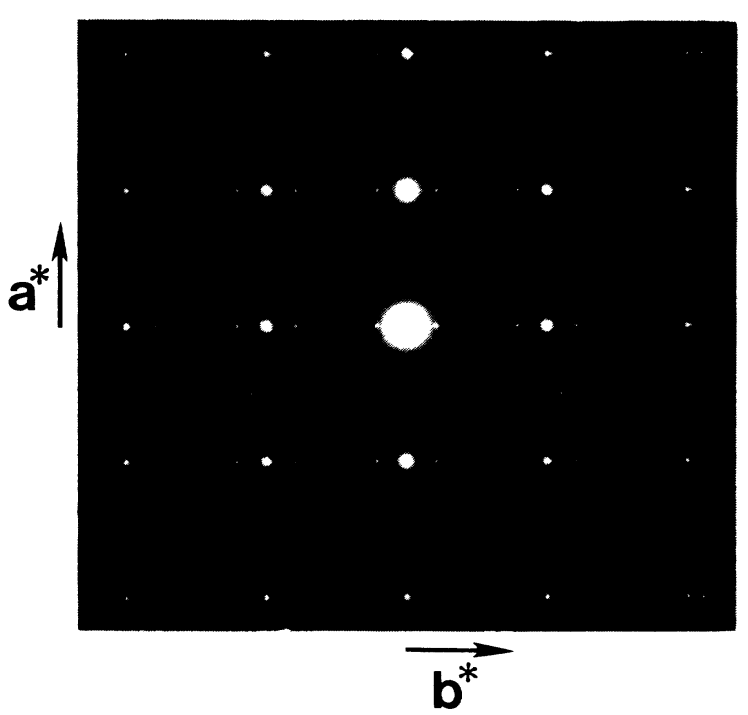

FIG. 2. Selected-area electron diffraction pattern of the Bi$\mathrm{Ca}-\mathrm{Sr}-\mathrm{Cu}$ oxide superconductor showing $h k 0$ diffraction spots. Strong spots correspond to $2.7 \times 2.7-\AA^{2}$ subcell, while superlattice reflections along $a^{*}$ and $b^{*}$ indicate spacings of 5.4 and $27.2 \AA$, respectively. techniques. Though the stoichiometry is similar to $\mathrm{CaCu}_{2} \mathrm{O}_{3}$, the unit cell and structure differ from those reported by Teske and Müller-Buschbaum for the pure calcium copper oxide. ${ }^{7}$ Standard indexing procedures yield an $F$-centered orthorhombic unit cell with $a$ $=11.328, b=12.774$, and $c=3.896 \AA$ for all three crystals. Striations and peak splittings characteristic of twinning were observed for these grains, however, and it is likely that the symmetry is monoclinic. A set of x-ray intensities has been collected; structure analysis of this phase is in progress.

A third minor phase, distinguished by a composition enriched in bismuth and lacking in copper, was observed by electron microanalysis in sample BCSCO- $a$. The composition, based on analyses of three different equant grains and normalized to one $\mathrm{Bi}$, is $\left(\mathrm{Ca}_{0.45} \mathrm{Sr}_{0.39}\right) \mathrm{BiO}_{3}-\delta$. Two poorly resolved electron diffraction patterns were obtained for this phase; one reveals an orthogonal net approximately $10.5 \times 3.0 \AA^{2}$ and the other shows a $9.5-\AA$ spacing with faint intermediate supercell reflections, possibly characteristic of a $19.0-\AA$ repeat. The structure of this phase may be related to strontium bismuth oxide $\mathrm{Sr}_{0.9} \mathrm{Bi}_{1.1} \mathrm{O}_{2.55}$, a tetragonal phase with $a=13.329$ and $c=4.257 \AA$, which was described by Guillermo et al. ${ }^{8}$ That phase displays characteristic spacings of $9.30 \AA$ (110) and $3.06 \AA$ (301).

The fourth phase-the superconducting compound - is abundant in all three samples. It is distinguished by

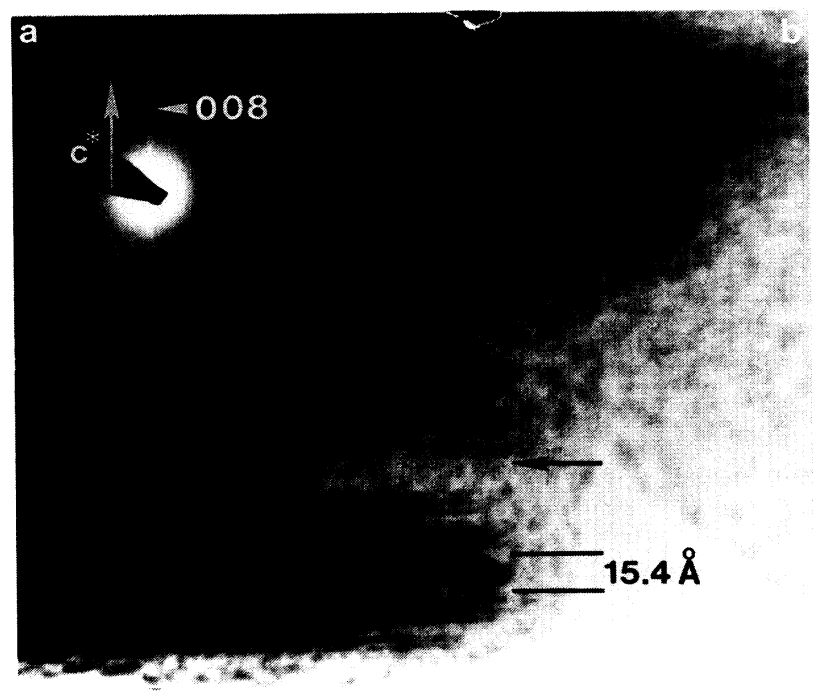

FIG. 3. (a) Selected-area electron diffraction pattern of the $00 l$ diffraction row, which is streaked but shows a strong 15.4$\AA$ periodicity. The 004 diffraction is indicated. (b) A highresolution image taken parallel to the layers shows the $15.4-\AA$ (002) spacing, with subspacings of $3.8 \AA$. The contrast of these layers differs, suggesting a possible ABAC-ABAC type stacking of perovskite and $\mathrm{Bi}_{2} \mathrm{O}_{2}$ units. A structural defect (arrowed) may correspond to a Bi-free region of $\mathrm{Ca}-\mathrm{Sr}-\mathrm{Cu}$ perovskite. 
a layered structure probably related to the class of layered bismuth compounds described by Aurivillius. ${ }^{9}$ These structures incorporate both perovskite-type layers and unusual $\mathrm{Bi}_{2} \mathrm{O}_{2}$ layers of bismuth in distorted fourcoordination. Electron microscopy reveals that grains possess a perfect basal (hereafter termed 001) cleavage, similar to that of clay minerals. The structure, therefore, must consist of a layered atomic arrangement with planes of weak bonding. Electron diffraction of (001) layers is facilitated by the tendency of almost every grain to lie flat on the holey-carbon film. Numerous $(h k 0)$ electron diffraction patterns were obtained (Fig. 2), all of which show a prominent perovskite-like $2.7 \times 2.7-\AA^{2}$ subcell and a distinctive $5.41 \times 27.2-\AA^{2}$ supercell. A few grains, lying near the edge of the grid, were oriented with (001) layers perpendicular to the grid. From these crystallites a stacking periodicity of $15.39 \AA$ was observed [Fig. 3(a)]. High-resolution images clearly reveal a four-layer structure with a $3.86-\AA$ subcell [Fig. 3(b)]. The image contrast of these layers suggests a possible ABAC-ABAC repeat pattern.

A single grain oriented with $a^{*}$ perpendicular to the grid yielded an electron density pattern corresponding to a $5.44 \times 30.78-\AA^{2}$ centered lattice with a five-repeat $(27.2 \AA)$ superlattice parallel to $b^{*}$ (Fig. 4). Highresolution images of this plane show the "bricklike" pattern characteristic of a centered structure, as well as numerous stacking faults and other defects. Many of these defects are probably associated with interfaces between perovskite and $\mathrm{Bi}_{2} \mathrm{O}_{2}$ modules, both of which

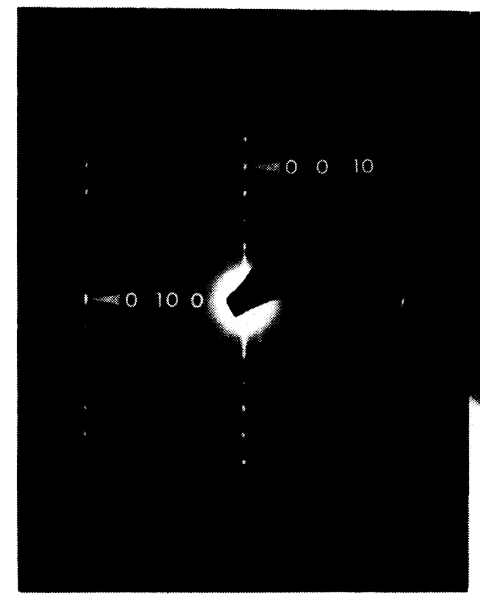

(a)

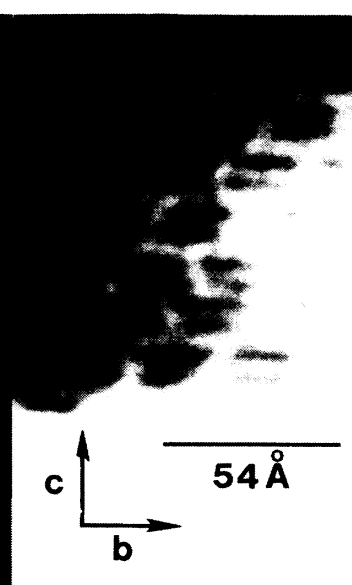

(b)
FIG. 4. (a) The selected-area $0 k l$ electron diffraction pattern is characterized by an $A$-centered $27 \times 31-\AA^{2}$ lattice. (b) A high-resolution image of the (100) plane reveals numerous stacking faults and defects as well as the $A$-centered layered structure. Though most of the structure appears to be orthogonal, locally inclined blocks may indicate a fine-scale twinning or may represent "monoclinic" regions of related but different structure. display approximately $3.8-\AA$ layer spacings.

Initial TEM cell parameters were used to index 26 lines in the complex x-ray powder pattern (Table I). The moderately strong 002 line at $15.4 \AA$ provides a useful marker for this phase. Orthorhombic lattice constants refined from powder diffraction data are $a$ $=5.410 \pm 0.003, b=5.439 \pm 0.005(\times 5)$, and $c=30.78$ $\pm 0.03 \AA$. These parameters are related to the simple cubic perovskite cell (a cube with $a=3.85 \AA$ ) by the ratios $\sqrt{2} \times 5 \sqrt{2} \times 8$.

The superconductor occurs in fine-grained masses of black, opaque flattened crystallites. The average diameter of these thin plates is less than $5 \mu \mathrm{m}$ and the thickness is usually less than $0.1 \mu \mathrm{m}$. Polished scanning electron microscopy mounts reveal that this phase forms a matrix of randomly oriented interlocking flakes in which the other single-crystal phases "float." This texture implies that the superconducting phase crystallized last, at a lower temperature than the other phases. The average composition of the superconductor was determined from fifteen analyses from three different samples. The ratio of cations approximates $2: 1: 2: 2$ for $\mathrm{Bi}: \mathrm{Ca}: \mathrm{Sr}: \mathrm{Cu}$. The average formula may be represented more precisely as $\mathrm{Bi}_{2}\left(\mathrm{Ca}_{0.39} \mathrm{Sr}_{0.56} \mathrm{Bi}_{0.05}\right)_{3} \mathrm{Cu}_{2} \mathrm{O}_{8+\delta}$. Note that bismuth

TABLE I. Powder $\mathrm{x}$-ray diffraction data for the Bi-Ca-Sr$\mathrm{Cu}-\mathrm{O}$ superconductor were obtained with filtered $\mathrm{Cu}$ radiation. Pure silicon (National Bureau of Standards standard reference material No. 640) was used as an internal standard.

\begin{tabular}{rrrccr}
\hline \hline$h$ & $k$ & $l$ & $d_{\text {obs }}$ & $d_{\text {calc }}$ & $I / I_{0}$ \\
\hline 0 & 0 & 2 & 15.66 & 15.39 & 12 \\
0 & 1 & 1 & 5.362 & 5.359 & 5 \\
0 & 0 & 8 & 3.857 & 3.847 & 23 \\
1 & 0 & 6 & 3.736 & 3.723 & 3 \\
1 & 1 & 3 & 3.593 & 3.594 & 41 \\
1 & 1 & 5 & 3.257 & 3.256 & 100 \\
0 & 0 & 10 & 3.085 & 3.080 & 24 \\
1 & 1 & 7 & 2.890 & 2.891 & 62 \\
2 & 0 & 0 & 2.709 & 2.705 & 91 \\
2 & 0 & 2 & 2.668 & 2.664 & 19 \\
0 & 0 & 12 & 2.556 & 2.565 & 18 \\
2 & 1 & 1 & 2.413 & 2.415 & 5 \\
2 & 0 & 8 & 2.214 & 2.213 & 4 \\
0 & 0 & 14 & 2.200 & 2.199 & 3 \\
2 & 0 & 10 & 2.033 & 2.032 & 26 \\
1 & 1 & 13 & 2.013 & 2.015 & 11 \\
2 & 1 & 9 & 1.978 & 1.977 & 4 \\
2 & 2 & 0 & 1.915 & 1.918 & 31 \\
2 & 2 & 4 & 1.862 & 1.862 & 9 \\
1 & 1 & 15 & 1.809 & 1.809 & 13 \\
0 & 3 & 3 & 1.787 & 1.786 & 3 \\
3 & 1 & 3 & 1.687 & 1.689 & 5 \\
3 & 1 & 5 & 1.648 & 1.649 & 13 \\
3 & 0 & 8 & 1.634 & 1.633 & 7 \\
3 & 1 & 7 & 1.595 & 1.595 & 13 \\
3 & 1 & 9 & 1.531 & 1.531 & 10 \\
\hline \hline
\end{tabular}


forms a solid solution with strontium and calcium in some structures. ${ }^{10}$ Analysis totals, based on oxides and the assumption of $\mathrm{Cu}^{+2}$ and $\mathrm{Bi}^{+3}$, total approximately $97 \%$. It is likely, therefore, that some copper or bismuth is in a higher oxidation state.

Composition varies considerably from grain to grain. The ratio of $\mathrm{Cu}+\mathrm{Bi}$ to $\mathrm{Ca}+\mathrm{Sr}$ is relatively constant at 1.00: $(0.68 \pm 0.02)$. The ratio of $\mathrm{Cu}$ to $\mathrm{Bi}$, however, ranges from $40: 60$ to $49: 51$, with most compositions closer to the latter value. The $\mathrm{Sr}: \mathrm{Ca}$ ratio ranges from $38: 30$ to $43: 25$, with an average value of $40: 28$. Some of these variations may result from microscopic inclusions of other phases or from secondary scattering from adjacent grains. It seems likely, however, that the superconducting phase has a variable composition with $\mathrm{Bi}-\mathrm{Ca}-\mathrm{Sr}$ solid solution. The numerous defects and stacking faults provide another mechanism for incorporating composition variation. These variations may account for the differences between the superconductivity behavior displayed by the three samples.

Our apparently new structure is closely related to the 22-K superconducting phase described by Michel et al. ${ }^{3}$ which has an approximate formula $\mathrm{Bi}_{2} \mathrm{Sr}_{2} \mathrm{Cu}_{2} \mathrm{O}_{7}$ and unit-cell parameters $5.32 \times 26.6 \times 48.8 \AA^{3}$. Both phases display the distinctive $b^{*}$ superlattice behavior, both have a prominent layered habit, and both possess long $c$ axis repeats. Our phase appears to be distinct, however, on the basis of differences in $c$-axis repeat, $x$-ray powder diffraction patterns, and compositions.

Detailed description of the complex 2122 structure will require single crystals or improved powder diffraction data, coupled with high-resolution imaging of the lattice by TEM. These studies are now in progress. Nevertheless, several structural inferences can be drawn from the compositional, powder x-ray, and TEM data in hand. A one-dimensional electron density profile based on the observed powder diffraction intensities of $00 \mathrm{l}$ reflections indicates a pronounced layering of cations in (001), with substantial deviations from an ideal perovskite metal arrangement. Bismuth appears to be concentrated in layers, perhaps similar to the $\mathrm{Bi}_{2} \mathrm{O}_{2}$ slabs of phases such as $\mathrm{BaBi}_{4} \mathrm{Ti}_{4} \mathrm{O}_{15}{ }^{9}$ Weak electron density associated with every fourth layer of the structure suggests interlayer regions of weak bonding. While it is tempting to speculate that copper and oxygen adopt the planar configuration common to the other known hightemperature oxide superconductors, there is not yet any direct evidence to support this supposition.

Superstructures parallel to the $a$ and $b$ axes may be the result of cation and oxygen ordering; therefore, chains of copper in square-planar coordination may be present. Note, however, that electron diffraction patterns reveal extensive streaking along $c^{*}$ and $b^{*}$, as well as spot splitting, twinning, stacking faults, and other defects that will likely disrupt linear structures. If these features affect superconductivity then annealing conditions and oxygen content should have an important effect on the temperature and sharpness of the superconducting transitions. The numerous structural defects provide a mechanism for generation of homologous structures (such as the Michel phase?) with slightly larger or smaller superstructures. It is likely that further study in related chemical systems will reveal additional superconducting compounds, leading to even higher $T_{c}$.

In spite of the fine-grained texture and high density of defects, the presumed copper-oxygen layers appear to be continuous over hundreds of unit cells. This layered structure may possess one significant advantage in terms of processing and applications. Fabrication of the superconductor by compression into planes could yield superconductor components with enhanced properties (e.g., critical current) in a specific plane. Similarly, fabrication by rolling could produce wires more flexible than those of ordinary ceramic materials.

Work at the Geophysical Laboratory was supported by National Science Foundation Grants No. EAR8419982, No. EAR8608941, and No. EAR8618602; at Johns Hopkins University by National Science Foundation Grant No. EAR8609227; at University of Houston by National Science Foundation Grant No. DMR8616539, NASA Grant No. NAGW-977, the Texas Center for Superconductivity, and the T.L.L. Temple Foundation.

${ }^{1}$ J. G. Bednorz and K. A. Müller, Z. Phys. B 64, 189 (1986).

${ }^{2}$ M. K. Wu, J. R. Ashburn, C. J. Torng, P. H. Hor, R. L. Meng, L. Gao, Z. J. Huang, Y. Q. Wang, and C. W. Chu, Phys. Rev. Lett. 58, 908 (1987).

${ }^{3}$ C. Michel, M. Hervieu, M. M. Borel, A. Grandin, F. Deslandes, J. Provost, and B. Raveau, Z. Phys. B 68, 421 (1987).

${ }^{4}$ C. W. Chu, J. Bechtold, L. Gao, P. H. Hor, Z. J. Huang, R. L. Meng, Y. Y. Sun, Y. Q. Wang, and Y. Y. Xue, Phys. Rev. Lett. 60, 941 (1988).

${ }^{5}$ P. H. Hor, R. L. Meng, Z. J. Huang, L. Gao, J. Bechtold, Y. Y. Xue, Y. Q. Wang, Y. Y. Sun, and C. W. Chu, to be published.

${ }^{6}$ C. L. Teske and H. Müller-Buschbaum, Z. Anorg. Allg. Chem. 379, 234 (1970).

${ }^{7}$ C. L. Teske and H. Müller-Buschbaum, Z. Anorg. Allg. Chem. 370, 134 (1969).

${ }^{8}$ R. Guillermo et al., Rev. Chim. Miner. 15, 153 (1978).

${ }^{9}$ B. Aurivillius, Ark. Kemi 1, 463, 499 (1950), and 2, 519 (1950).

${ }^{10} \mathrm{P}$. Conflant, J. C. Boivin, and D. Thomas, J. Solid State Chem. 35, 192 (1980). 


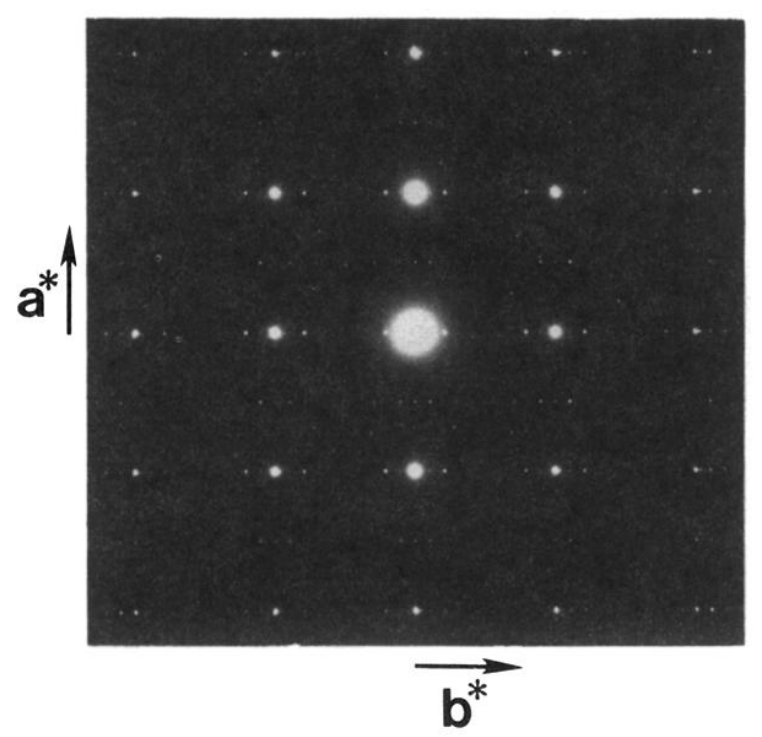

FIG. 2. Selected-area electron diffraction pattern of the Bi$\mathrm{Ca}-\mathrm{Sr}-\mathrm{Cu}$ oxide superconductor showing $h k 0$ diffraction spots. Strong spots correspond to $2.7 \times 2.7-\AA^{2}$ subcell, while superlattice reflections along $a^{*}$ and $b^{*}$ indicate spacings of 5.4 and $27.2 \AA$, respectively. 


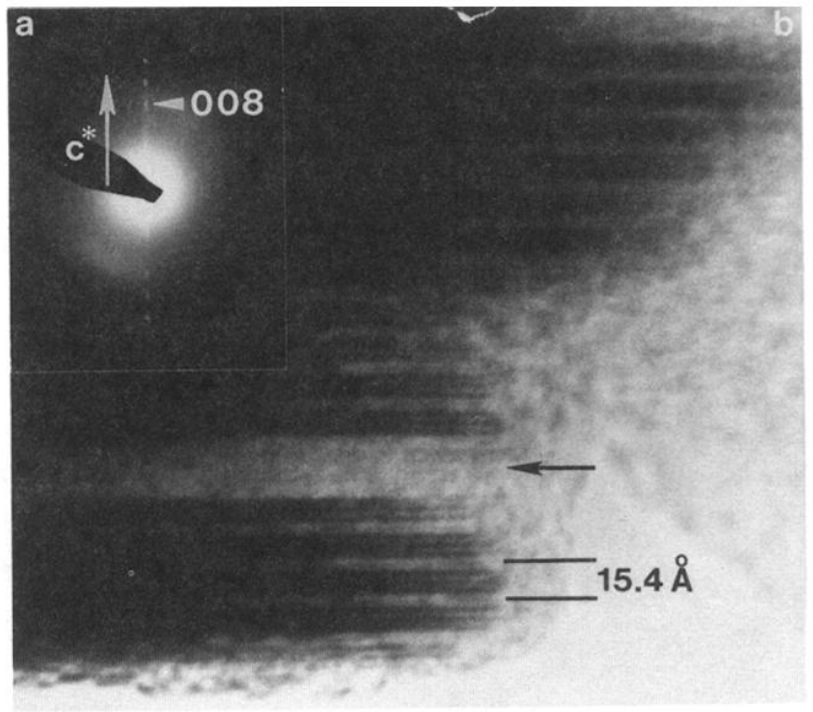

FIG. 3. (a) Selected-area electron diffraction pattern of the $00 l$ diffraction row, which is streaked but shows a strong 15.4$\AA$ periodicity. The 004 diffraction is indicated. (b) A highresolution image taken parallel to the layers shows the $15.4-\AA$ (002) spacing, with subspacings of $3.8 \AA$. The contrast of these layers differs, suggesting a possible ABAC-ABAC type stacking of perovskite and $\mathrm{Bi}_{2} \mathrm{O}_{2}$ units. A structural defect (arrowed) may correspond to a $\mathrm{Bi}$-free region of $\mathrm{Ca}-\mathrm{Sr}-\mathrm{Cu}$ perovskite. 


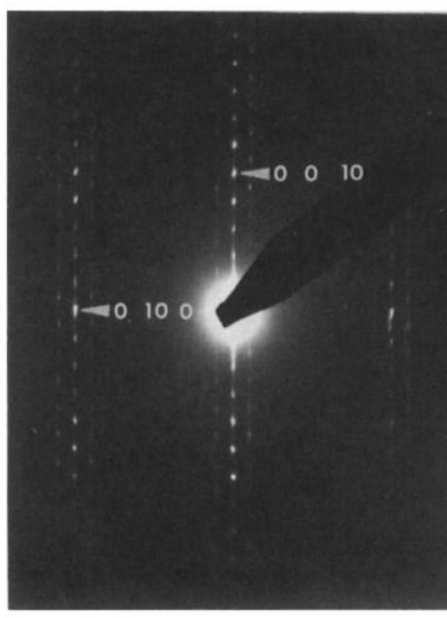

(a)

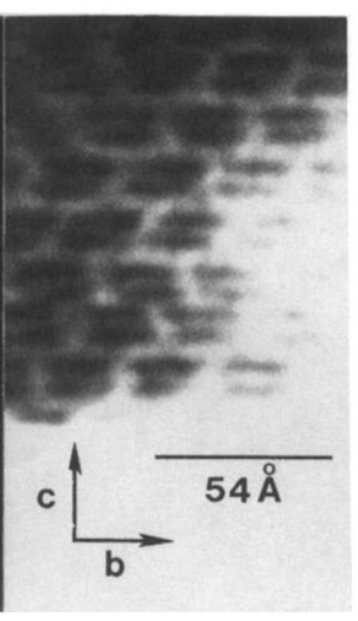

(b)

FIG. 4. (a) The selected-area $0 \mathrm{kl}$ electron diffraction pattern is characterized by an $A$-centered $27 \times 31-\AA^{2}$ lattice. (b) A high-resolution image of the (100) plane reveals numerous stacking faults and defects as well as the $A$-centered layered structure. Though most of the structure appears to be orthogonal, locally inclined blocks may indicate a fine-scale twinning or may represent "monoclinic" regions of related but different structure. 